\title{
TREATMENT OF CHRONIC ASTHMA
}

\author{
John Rees, John Price
}

\section{$\beta$-stimulants}

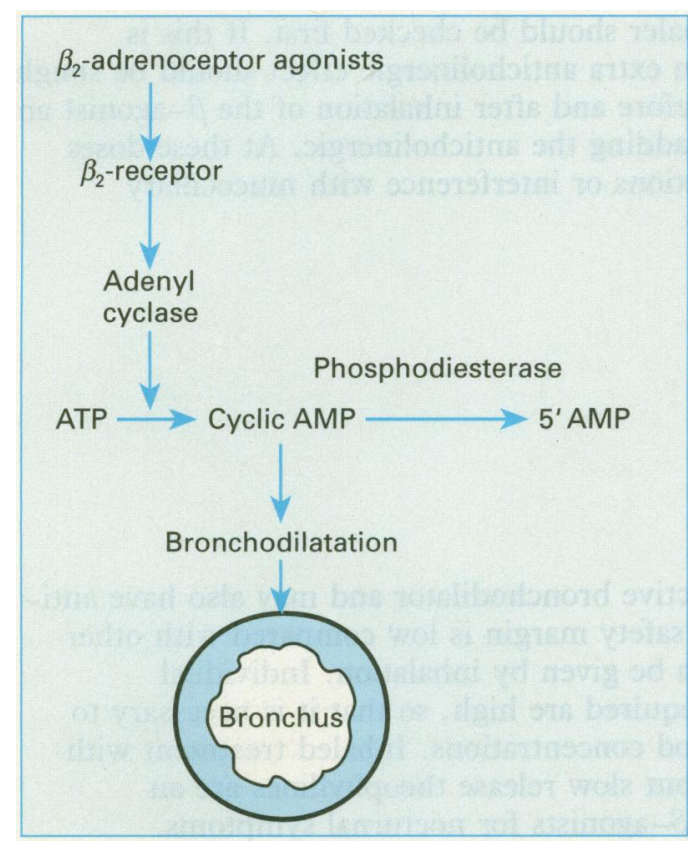

The first line of treatment of mild intermittent asthma is one of the selective $\beta_{2}$-stimulants taken by inhalation. $\beta$-stimulants are the most effective bronchodilator in asthma. They start to work quickly_salbutamol and terbutaline take effect within 15 minutes and last for four to six hours. If more than one daily dose is usually required then additional treatment must be considered. The dose response varies among patients as does the dose that will produce side effects, such as tremor. Patients should be taught to monitor their inhaler use and to understand that if they need it more or its effects lessen, these are danger signals. They indicate deterioration in asthmatic control and the need for further treatment.

Some patients worry that $\beta$-stimulants may become slightly less effective with time, particularly if the dose is high. There is little evidence of appreciable tachyphylaxis for the airway effects in asthmatics. If it exists it is a minor effect that is quickly reversed either by stopping the treatment temporarily or by taking corticosteroids. Tremor, palpitations, and muscle cramps may occur but are rarely troublesome if the drug is inhaled and these adverse effects outside the lung often become less of a problem with continued treatment.

The regular use of $\beta$-stimulants has in some studies been associated with increased bronchial reactivity, worsening asthma control, and accelerated decline of lung function. When the steps in the guidelines are followed, however, $\beta$-stimulants are not used regularly unless needed for control of symptoms.

\section{Long acting $\boldsymbol{\beta}$-stimulants}

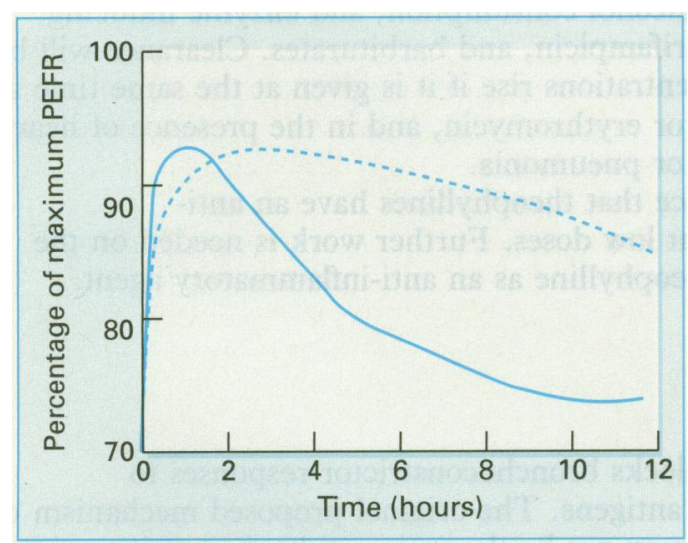

Bronchodilator response to oral salbutamol $200 \mu \mathrm{g}$ (solid line) and inhaled salmeterol $50 \mu \mathrm{g}$ (broken line).

Long acting preparations of oral $\beta$-agonists have been available for some years. They are effective in nocturnal asthma but are associated with more adverse effects than inhaled agents. The oral agent bambuterol, a prodrug of terbutaline, provides a newer longer acting alternative.

Long acting inhaled preparations are now available. They have an action that lasts at least 12 hours and can be used twice daily, or just at night if they are to prevent nocturnal problems. The protective effect against challenge may decline with prolonged use but the degree and duration of bronchodilator effect is maintained.

The precise place of the long acting agents is uncertain. There is a worry that their effectiveness and prolonged protection might mean that anti-inflammatory agents are neglected. This might allow underlying inflammation to continue so that a sufficient challenge might still lead to severe narrowing of the airway.

At present in asthma the long acting $\beta$-stimulants should be used only as an addition to inhaled corticosteroids. There is a debate about the dose of steroids achieved before the addition of long acting inhaled $\beta$-agonists. Some guidelines suggest a dose of 1000-2000 $\mu \mathrm{g}$ daily; others that are worried about the adverse effects of higher doses of inhaled corticosteroids suggest that drugs such as salmeterol should be added if control is inadequate with doses of corticosteroid below $1000 \mu \mathrm{g}$. Clinical studies have shown the effectiveness and safety of this approach. 


\section{Anticholinergic bronchodilators}

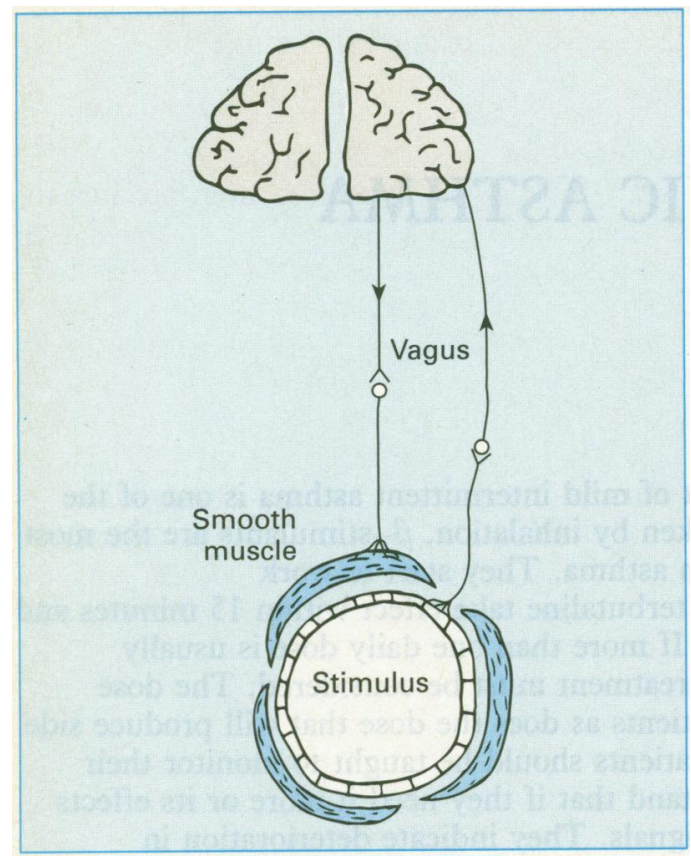

Anticholinergic agents block vagal efferent stimulation of bronchial smooth muscle.

\section{Methylxanthines}

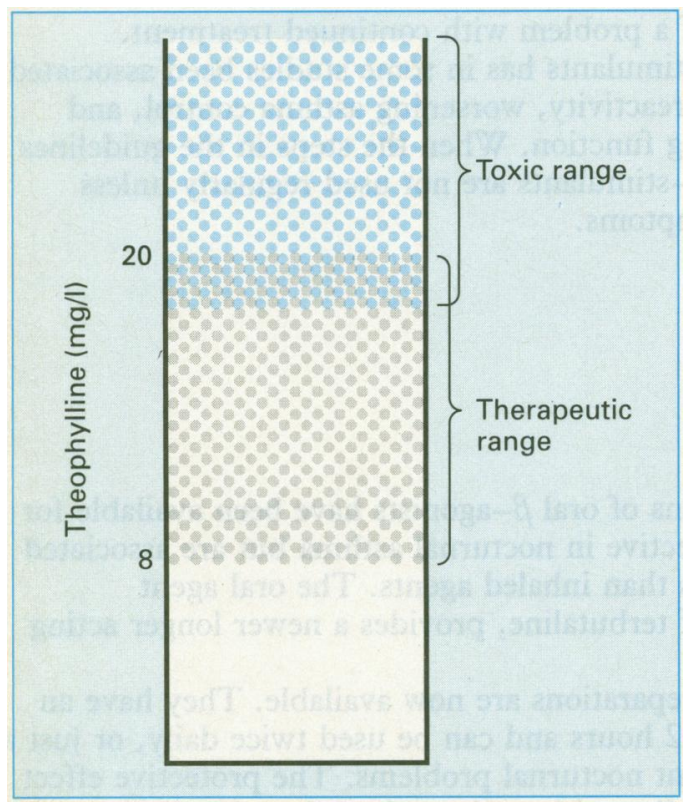

There is no safety margin between therapeutic and toxic ranges with theophylline.
Ipratropium bromide blocks the cholinergic bronchoconstrictor effect of the vagus nerve. Oxitropium bromide has a longer action, which makes it suitable for use two or three times a day. Anticholinergics are most effective in very young children and in older patients, and are generally more effective than $\beta$-stimulants in chronic bronchitis and emphysema. In most cases of asthma anticholinergic agents are less effective than $\beta$-stimulants, but they may supplement their effect if reversibility is incomplete. Anticholinergics take second place as bronchodilators in asthma unless tremor or tachycardia are troublesome side effects. If the response to a $\beta$-stimulant is inadequate then the technique of using the inhaler should be checked first. If this is satisfactory, evidence of an extra anticholinergic effect should be sought by measuring peak flow before and after inhalation of the $\beta$-agonist and then 30-60 minutes after adding the anticholinergic. At these doses there is no drying of secretions or interference with mucociliary clearance.
Theophylline is an effective bronchodilator and may also have antiinflammatory actions. Its safety margin is low compared with other bronchodilators which can be given by inhalation. Individual differences in the doses required are high, so that it is necessary to monitor treatment by blood concentrations. Inhaled treatment with $\beta$-agonists is preferable, but slow release theophyllines are an alternative to long acting $\beta$-agonists for nocturnal symptoms. Absorption of aminophylline from suppositories is much less predictable and they are best avoided.

The commonest side effects of theophylline are nausea, vomiting, and abdominal discomfort, but headache, malaise, fast pulse rate, and fits also occur, sometimes without early warning from gastrointestinal symptoms. The dose of theophylline should start at around $7 \mathrm{mg} / \mathrm{kg} /$ day in divided doses, and build up. All patients taking theophylline should have their peak concentrations monitored and doses adjusted until they are between 8 and $18 \mathrm{mg} / 1(40-90 \mu \mathrm{mol} / \mathrm{l})$. Above $20 \mathrm{mg} / \mathrm{l}$ toxic effects are unacceptably high, although $15 \%-30 \%$ of patients will have gastrointestinal effects with smaller doses. Theophylline clearance is increased by smoking, alcohol consumption, and enzyme inducing drugs such as phenytoin, rifampicin, and barbiturates. Clearance will be decreased and blood concentrations rise if it is given at the same time as cimetidine, ciprofloxacin, or erythromycin, and in the presence of heart failure, liver impairment, or pneumonia.

There is in vitro evidence that theophyllines have an antiinflammatory effect even at low doses. Further work is needed on the clinical use of low dose theophylline as an anti-inflammatory agent.

\section{Sodium cromoglycate and nedocromil sodium}

Must be used regularly for at least 4-8 weeks before being dismissed as ineffective.
Sodium cromoglycate blocks bronchoconstrictor responses to challenge by exercise and antigens. The original proposed mechanism of stabilisation of mast cells may not be the main mechanism of its action in asthma. It can be used as the first line prophylactic agent if control of asthma requires more than an occasional inhalation of $\beta$-agonist, and success is most likely with young atopic asthmatic patients but may occur at any age. Sodium cromoglycate used to be given as a dry powder in Spincaps but metered dose inhalers delivering $5 \mathrm{mg} /$ 


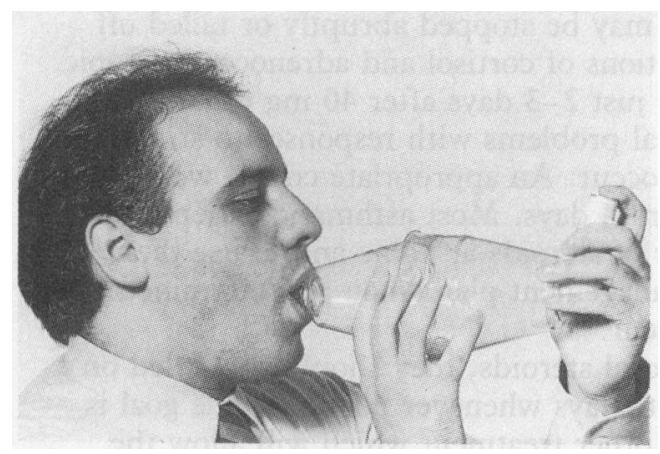

The large volume spacers overcome problems with coordination of inhaler firing and inspiration. They reduce oropharyngeal deposition of aerosol and improve delivery to the lung.

\section{Inhaled corticosteroids}

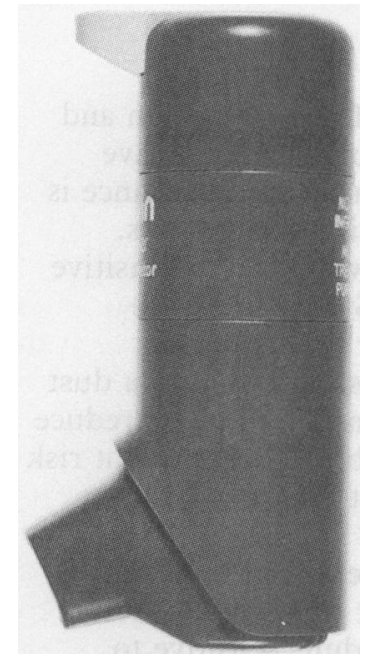

The autohaler device is triggered by inspiratory airflow and is available for $\beta$-agonists, anticholinergics and corticosteroids.

\section{Side effects of inhaled corticosteroids}

Established:

- Oropharyngeal candida

- Dysphonia

- Irritation and cough

Suggested at high dose:

- Adrenal suppression

- Reduced growth in children

- Purpura and thinning of skin

- Osteoporosis

- Cataract actuation are more convenient and just as effective. Occasionally patients develop reflex bronchoconstriction in response to the irritant effects of the dry powder, and if they do, they should try changing to a metered dose inhaler or using a dose of inhaled $\beta$-agonist $10-15$ minutes earlier.

Other adverse reactions to sodium cromoglycate are rare. Other mast cell stabilisers have been disappointing, possibly because of the additional effects of cromoglycate. The oral agent ketotifen produces drowsiness in $10 \%$ of patients and has little activity.

Cromoglycate should not be dismissed as ineffective until it has been tried for at least 4-8 weeks and it must be used regularly. It has no place in the treatment of acute exacerbations of asthma and may even increase narrowing of the airways by its irritant effect.

Nedocromil sodium has the same properties as sodium cromoglycate but may have an additional anti-inflammatory effect on the airway epithelium. It is probably more useful than sodium cromoglycate in older patients although it is less effective than inhaled corticosteroids in most subjects. It may be tried as a first line in patients with mild disease or as an addition to inhaled corticosteroids when control of symptoms is inadequate.

Steroids may be given by metered dose inhaler or dry powder devices, and the dose should be adjusted to give optimum control. The two commonest inhaled steroids, beclomethasone dipropionate and budesonide, are roughly equivalent in dose. The newer agent fluticasone seems to have less systemic effects at the same dose and it can be used at half the dose of beclomethasone dipropionate with the same therapeutic effect.

Inhaled steroids may be given twice daily with no problems apart from occasional oropharyngeal candidiasis or a husky voice until a daily dose above the equivalent of $1000 \mu \mathrm{g}$ beclomethasone dipropionate is reached. At higher doses there may be biochemical evidence of. suppression of the hypothalamic-pituitary-adrenal axis, even with inhaled steroids, but this is not a particular problem in adults.

With doses of more than $1000 \mu \mathrm{g}$ daily of budesonide or beclomethasone there are metabolic effects including an increase in the concentration of osteocalcin, a marker of increased bone turnover. There is some evidence of skin thinning and purpura even in patients who have not had appreciable doses of oral steroids. Doses over $2000 \mu \mathrm{g}$ daily are not often used but when necessary nebulised budesonide may be a convenient strategy. A large volume spacer should be used at doses above $800 \mu \mathrm{g}$ daily to reduce the pharyngeal deposition of metered dose inhalers.

Doses of inhaled steroids should be taken regularly to be effective. There is evidence that doubling the regular dose when an upper respiratory infection develops reduces the risk of problems from an exacerbation of asthma.

The main difficulties in the use of inhaled corticosteroids are the patients' worries about the use of steroids and the difficulties of ensuring that patients take regular medication even when they are well. These problems are increased by the move to use inhaled corticosteroids earlier in asthma and to try to achieve a level of control that is free of symptoms.

There is a suggestion that control is achieved more easily by the use of a high dose of inhaled steroids at the start then a reduction as control is achieved. When asthma is under control the next decision is how long to maintain the inhaled steroids. The dose should be reviewed regularly and if doses above $1000 \mu \mathrm{g}$ daily have been necessary these should be reduced when possible. Most physicians like to have complete asthma control for 6-12 months before trying to stop prophylaxis completely.
Occasional asthmatic patients have to take long term oral corticosteroids but this should be only after the failure of vigorous treatment with other drugs, and the symptoms or risks of the disease must be balanced against the adverse effects of long term treatment with oral corticosteroids. It is important to remember that, in contrast,
Long term corticosteroids should be used only if other drugs have failed.

\section{Oral corticosteroids}




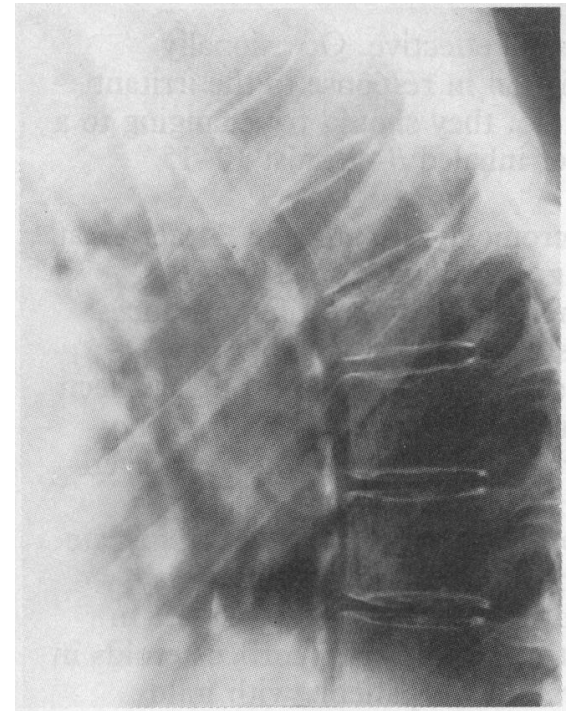

Osteoporotic collapse of a thoracic vertebra in a patient taking oral steroids. short courses of oral steroids for exacerbations of symptoms and inhaled steroids have few serious problems.

Short courses of oral steroids may be stopped abruptly or tailed off over a few days. Low concentrations of cortisol and adrenocorticotropic hormone (ACTH) are found for just 2-3 days after $40 \mathrm{mg}$ prednisolone daily for three weeks, but clinical problems with responses to stress or exacerbations of asthma do not occur. An appropriate course would be 25-40 mg prednisolone daily for 14 days. Most asthmatic patients can be taught to keep such a supply of steroids at home and to use them according to their individual management plan when predetermined signs of deteriorating control occur.

If patients require long term oral steroids, they should be settled on a regimen of treatment on alternate days whenever possible. The goal is always to establish control with other treatment which will allow the discontinuation of the oral steroids. Inhaled steroids in moderate to high doses should be maintained to keep the oral dose as low as possible. Alternative preparations such as ACTH and triamcinolone are less flexible and give no appreciable benefit in terms of adrenal suppression.

A small proportion of asthmatic patients are fully or partially resistant to corticosteroids. They form a particularly difficult group to treat.

\section{Desensitisation and avoidance of allergens}
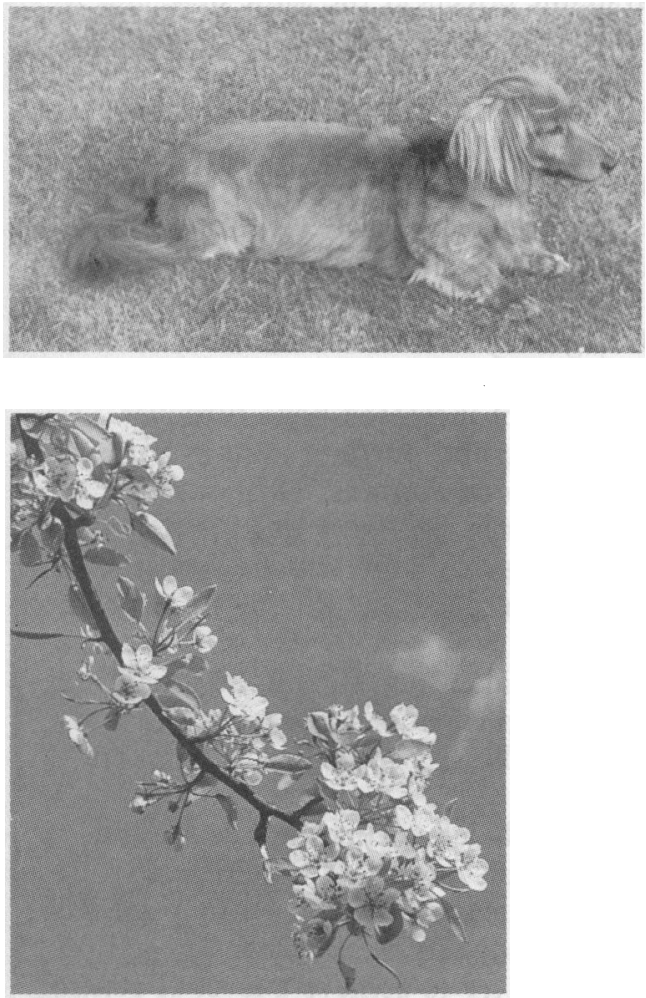

\section{Other drug treatments}

Preparations which combine a prophylactic agent and a short acting bronchodilator do not fit into care guidelines. Inhaled bronchodilators should be used as necessary.
As discussed in chapter 4 , the results of trials of desensitisation and avoidance of allergens have been disappointing. Some patients have obvious precipitating factors-in particular, animals - and avoidance is helpful, but there are usually other unknown precipitating factors. More common are patients with reactive airways who are also sensitive to pollens, house dust mite, and other allergens. Such stimuli are almost impossible to avoid completely in everyday life, although symptoms improve with rigorous measures such as admission to a dust free environment in hospital. Nevertheless it is sensible to try to reduce the exposure to known allergens as much as possible. In children at risk of asthma it may be particularly important to limit their exposure to potential allergic problems.

There is some evidence that desensitisation is beneficial in patients with asthma who are sensitive to pollens, and that repeated courses increase the improvement. Controlled studies in adults sensitive to house dust mites have shown no benefit from desensitisation. Several studies in children have suggested some benefit, but these were highly selected patients, and it is unusual to find asthmatic patients with a single sensitivity. The degree of control produced by desensitisation can usually be achieved with simple, safe, inhaled drugs.

There is little sound evidence to support desensitisation to other agents in asthmatic patients. In particular, cocktails produced from the results of skin tests or radioallergosorbent tests are not a valid form of treatment. Local reactions to desensitising agents are common and more generalised reactions and even death can occur. Most deaths are related to errors in the injection schedule and inadequate supervision after injections. Desensitisation should be undertaken only where appropriate facilities for resuscitation are available.

One area where desensitisation is appropriate is in sensitivity to insect venom which results in anaphylaxis rather than asthma. Aspirin induced asthma may respond to careful oral desensitisation.

Some fixed dose combinations are available for the treatment of asthma, but they have little to commend them. Combinations of bronchodilators may be used when such treatment has been shown to be appropriate in drug and in dose. This is unusual in asthma.

Combinations of bronchodilator and prophylactic drug do not fit easily into most guidelines for the treatment of asthma. It has been suggested that they may improve compliance with the prophylaxis, but this is not certain. They take away flexibility of treatment. Several oral combinations are available, often with small doses of theophylline, ephedrine, and barbiturate. There is no indication for such preparations. 


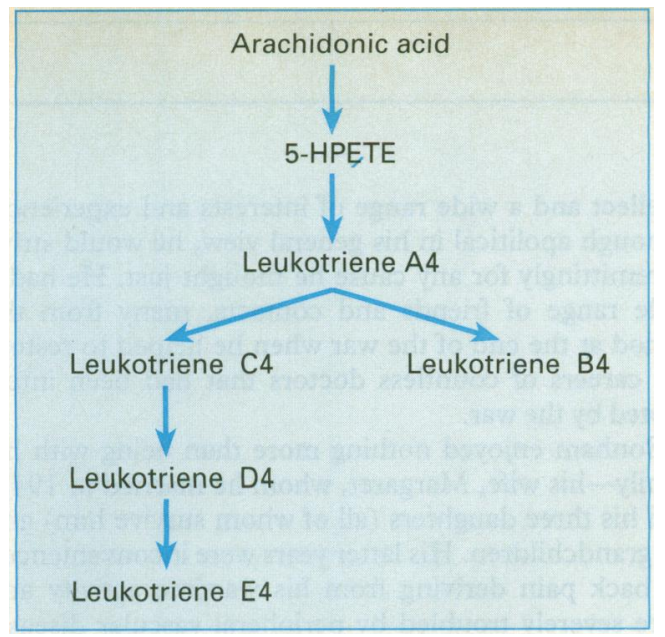

Leukotriene antagonists are being developed and may act as alternative prophylactic agents in asthma.
Other drugs such as $\alpha$-stimulants, antihistamines, and calcium antagonists may have some effect, but have not found a role in treatment. Newer antagonists to leukotrienes, platelet activating factor, and other mediators are under trial.

When large doses of corticosteroids are necessary, or in patients who fail to respond to steroids, other agents have been tried. Methotrexate, gold, and cyclosporin have shown evidence of benefit, mainly in reducing the dose of steroids necessary for control. All are associated with substantial potential side effects.

\section{Other treatments}

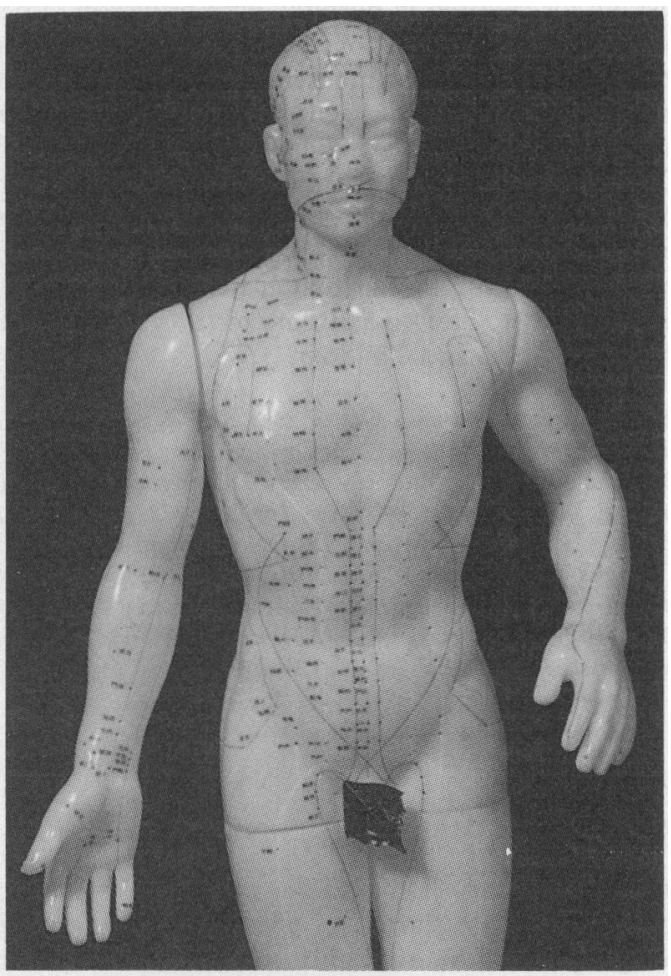

There have been dramatic claims for the benefit of yoga, hypnosis, and acupuncture but few have been confirmed in controlled trials. Relaxation may help to reduce the anxiety and hyperventilation which can exacerbate asthma. Such techniques may be useful in dealing with exacerbations. Patients' worries about the future course of asthma and its dangers should be explored and dealt with.

Ionisation of inspired air may have a small effect on lung function and may attenuate the response to exercise, but such effects are minimal and not achieved by home ionisers. Some herbal remedies may contain useful ingredients which need investigation. Some contain conventional agents which are not standardised, however, and some even contain corticosteroids and have produced the expected side effects.

This article is taken from the new edition of the ABC of Asthma

\section{ONE HUNDRED YEARS AGO}

\section{SCHOOL SLATES}

In one of a series of popular health lectures recently delivered at Perth, Dr. Ferguson drew attention to a very common practice among school children which may affect the spread of tuberculosis. He says that, in certain schools, slates are handed to the children, and that there is not the slightest attempt made to ensure that each child shall have the same slate time after time. Now the first thing the child does is to clean the slate, which is done in a primitive method, by means of the finger wetted with saliva. In this process the finger travels many times from mouth to slate, and vice versa, and thus conveys to the mouth any material which may happen to be upon the slate. What this material may be depends largely on the health of the child who previously used the slate. Dr. Ferguson's special point was that tuberculosis might thus be transmitted from child to child; but it is even more obvious that such a disease as diphtheria might be diffused in this manner. Many people doubt the educational advantage of using slates at all; certainly if they are used every child should have his own, as is the rule in some American schools.

(BMF 1895;i:771.) 